\title{
A Terahertz Vortex Beam Emitter with Tunable Topological Charge and Harmonic Excitation
}

\author{
Zi-Wen Zhang, Chao-Hai Du, Senior Member, IEEE, Juan-Feng Zhu, Feng-Yuan Han, Fan-Hong Li, \\ Zi-Chao Gao, Liang Zhang, Senior Member, IEEE, Adrian W. Cross, Member, IEEE, Pu-Kun Liu, \\ Senior Member, IEEE
}

\begin{abstract}
Terahertz (THz) vortex beams carrying orbital angular momentum (OAM) with high purity and tunable topological charge (TC) will undoubtedly bring extraordinary capacities for advanced imaging or communication systems. We propose a convenient and efficient method to generate a tunable and broadband vortex beam that is excited by superradiant Smith-Purcell radiation (SSPR) on a helical grating. This scheme fully explores the advantages of natural broadband evanescent wave carried by the electron beam and the Bloch's theorem regulated helical periodic systems. An explicit relation is established between the index of the spatial harmonic wave on the grating and the topological charge of the vortex beam. The electron energy in the SSPR can be customized to ignite the specific spatial harmonics and manipulate the OAM beam, accordingly. The separated radiation region also promises the high purity of the OAM spectrum. The harmonic excitation reduces the communality between the wavelength and the device size and alleviates the difficulty of device fabrication in the THz band. The proposed vortex scheme can not only be handily scaled to microwave and mid-infrared regions, but also bring possibilities to applications based on compact tunable vortex beam sources.
\end{abstract}

Index Terms-Vortex beams, orbital angular momentum, terahertz radiation, harmonic operation, vacuum electronic device.

\section{INTRODUCTION}

$\mathrm{S}_{1}^{\mathrm{N}}$ INCE the optical vortex beam was discovered by Allen in 1992 [1], it has aroused widespread interest resulting in extensive research in this area. It is also known as the orbital angular momentum (OAM) beam according to the photons of vortex beams possessing OAM in addition to spin angular momentum (SAM) [2]. Due to the physical characteristic of OAM, e.g., interaction with matter and topological protection [3], the optical vortex has been applied to numerous fields, such as optical manipulation, imaging, sensing, and communication

This work was supported in part by the National Natural Science Foundation of China under contracts 61861130367, NSAF-U1830201, and 61971013. It was also supported in part by the National Key Research and Development Program under contract 2019YFA0210203, and Newton Advanced Fellowship from Royal Society (NAF/R1/180121), United Kingdom.

Zi-Wen Zhang, Chao-Hai Du, Juan-Feng Zhu, Feng-Yuan Han, Fan-Hong $\mathrm{Li}, \mathrm{Zi}-\mathrm{Chao} \mathrm{Gao}$, and Pu-Kun Liu are with the State Key Laboratory of Advanced Optical Communication Systems and Networks, Department of Electronics, Peking University, Beijing 100871, China (e-mail: duchaohai@pku.edu.cn).

Liang Zhang and Adrian W. Cross are with the Department of Physics, SUPA, University of Strathclyde, Glasgow G4 0NG, Scotland, UK.
[4-8], etc. The vortex in the terahertz (THz) frequency band is also appealing due to many potential applications, such as $\mathrm{THz}$ communication, super-resolution imaging, spectroscopic probes, electron acceleration, and biomedical engineering [913], etc. Therefore, the generation of the $\mathrm{THz}$ vortex beams has become an attractive topic in the past decade, and many excellent research works have emerged. A dielectric spiral phase plate can directly and effectively generate the $\mathrm{THz}$ vortex, which provides confinement of the azimuthal spiral phase and converts the incident Gaussian beam into a vortex [14]. A metasurface with circular subwavelength slits was proposed to convert the SAM of the incident $\mathrm{THz}$ waves to the OAM and to generate the THz vortex [15]. Another method of generating broadband $\mathrm{THz}$ vortex beams is by manipulating radially polarized beams [16]. Metasurfaces composed of the V-shaped unit cells or complementary split rings are designed to produce the $\mathrm{THz}$ vortices via precisely controlling the geometric parameters of the elements [17]. Furthermore, silicon binary diffractive optical elements [18], liquid crystals [19], and ZnTe cubic crystal [20] have also been proposed to generate the $\mathrm{THz}$ vortices.

However, in all these methods, once the structures are determined, the OAM of the resulting THz vortex beam cannot be tuned easily. They also require existing $\mathrm{THz}$ beams to be manipulated and regulated. Due to the lack of suitable devices and the diffraction of the waves in the $\mathrm{THz}$ band, it is inconvenient to convert the incident Gaussian beams into high-purity $\mathrm{THz}$ vortex beams [21]. Moreover, as for metasurface-based $\mathrm{THz}$ vortex beams, the operation bandwidth is relatively narrow in nature because they are implemented based on the principle of resonance [22]. Therefore, there is an urgent need to explore a simple method to generate broadband $\mathrm{THz}$ vortex beams with tunable OAM.

Similar to the interest in OAM, Smith-Purcell radiation (SPR) also intrigues scientists and engineers in recent years and has been employed to achieve various functional sources [23-26]. In 2019, the helix tape is utilized to generate the vortex SPR for the first time with two possible values $( \pm 1)$ of topological charge (TC), while the possibility of generating the higherorder topological charge (i.e., $\pm 2, \pm 3, \ldots$ ) is not discussed [27]. In 2020, inspired by the hologram plane, the fork structure is introduced into the conventional grating to emit the vortex SPR with the higher-order topological charge [28], whereas the bunched sheet-electron beam employed is challenging to be 


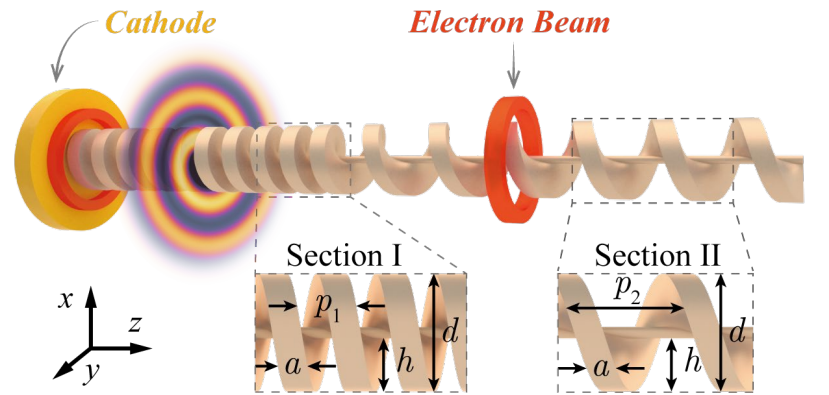

Fig. 1. Configuration of the double-section metallic helical grating. The periods of section-I and II are $p_{1}$ and $p_{2}\left(p_{2}=2 p_{1}\right)$, respectively. The width is $a$, the depth is $h$, and the diameter of the cylinder is $d$. The parameters above are shown in Table I. The hollow electron beam moves along the surface of the grating.

controlled in practice $[29,30]$.

This paper aims to demonstrate that the barrier of generating the THz vortex beam can be removed by superradiation SmithPurcell radiation (SSPR). In 1998, J. Urata et al. stated that SSPR could be generated once the interaction between the direct current (DC) electron beam and the surface wave is sufficiently large, and the radiation intensity was greatly enhanced at the specific harmonics [31]. Since then, $\mathrm{THz}$ sources based on SSPR were extensively studied and reported [32-38]. In this work, an emitter based on the helical grating integrated with SSPR is proposed to generate the $\mathrm{THz}$ vortex beams with tunable OAM. It is demonstrated in theory that every spatial harmonic of the scattered field carries the OAM corresponding to the spatial harmonic order when the evanescent waves carried by the electron beam are scattered in the helical periodic system. Based on a well-designed double-section helical grating, the vortex beams with disparate OAM will be generated once the wave vectors of the abovementioned spatial harmonics match those in free space. By switching the operation status between the second and third wave harmonic, the emitter can generate the $\mathrm{THz}$ vortex beam with broad tuning bandwidth and tunable TC. To further facilitate the feasibility, a section of the cylindrical grating is utilized to prebunch the DC electron beam. The scheme introduced in this work is compact and will benefit the development and applications of the $\mathrm{THz}$ vortex beam.

\section{OAM IN HELICAL PERIODIC SYSTEM}

The vortex beams, as electromagnetic waves with a helical wavefront and a singularity in phase, have the phase factor of $\exp (i \ell \varphi)$, where $\ell$ represents the TC surrounding the phase singularity, and $\varphi$ denotes the azimuthal coordinate in the cylindrical system. Thereby, in addition to the SAM related to polarization, each photon of the vortex beam also possesses OAM related to the wavefront, which can be quantized as $\ell \hbar$. Therefore, the TC can be used to distinguish different vortex beams. The model structure of the helical periodic system is shown in Fig. 1, which consists of two sections of the helical grating. Particularly, the period of section-II is twice that of section-I. According to Bloch's theorem, the scattered field $E_{z}$ of a time-harmonic evanescent wave carried by the electron beam in a right-hand metallic helical periodic system is
TABLE I

PARAMETERS OF THE HELICAL GRATING

\begin{tabular}{ll}
\hline \hline Parameter & Size $(\mathrm{mm})$ \\
\hline$p_{1}$ & 0.30 \\
$p_{2}$ & 0.60 \\
$a$ & 0.15 \\
$h$ & 0.27 \\
$d$ & 0.60 \\
\hline \hline
\end{tabular}

given by

$$
\begin{aligned}
& E_{z}(r, \varphi, z, t)=u(\varphi, z) R(r) \times e^{-j k_{z} z} e^{-j \omega t}, \\
& u(\varphi, z)=u(\varphi+2 \mathcal{N} \pi, z+\mathcal{N} p), \mathcal{N} \in \mathbb{Z},
\end{aligned}
$$

where $u(\varphi, z)$ is a periodic function consistent with the periodicity of the helical system, $k_{z}$ is the wavenumber in the $z$-direction, $R(r)$ is a function of the radius $r$, and $p$ is the pitch. By carrying out the Fourier expansion of $u(\varphi, z)$ relating to $\varphi$ and $z$ in (1), $u(\varphi, z)$ can be written as a Fourier series by separation of variables:

$$
\begin{aligned}
u(\varphi, z) & =\Phi(\varphi) \zeta(z) \\
& =\sum_{\ell=-\infty}^{\infty} \mathcal{A}_{\varphi}^{\ell} e^{j \ell \varphi} \sum_{\kappa=-\infty}^{\infty} \mathcal{A}_{z}^{\kappa} e^{j \kappa \frac{2 \pi}{p} z},
\end{aligned}
$$

where the coefficient $\mathcal{A}_{\varphi}^{\ell} \cdot \mathcal{A}_{z}^{\kappa}(\ell, \kappa \in \mathbb{Z})$ can be calculated by

$$
\mathcal{A}_{\varphi}^{\ell} \cdot \mathcal{A}_{z}^{\kappa}=\int_{0}^{2 \pi} \int_{0}^{p} u(\varphi, z) e^{-j n \varphi} e^{-j m \frac{2 \pi}{p} z} d z d \varphi
$$

Since the function $u(\varphi, z)$ is expressed using two periodic functions, the effectiveness of (4) should be simply demonstrated according to (3):

$$
\int_{0}^{2 \pi} \int_{0}^{p} \sum_{\ell=-\infty}^{\infty} \mathcal{A}_{\varphi}^{\ell} e^{j \ell \varphi} \sum_{\kappa=-\infty}^{\infty} \mathcal{A}_{z}^{\kappa} e^{j \kappa \frac{2 \pi}{p} z} e^{-j n \varphi} e^{-j m \frac{2 \pi}{p} z} d \varphi d z=\mathcal{A}_{\varphi}^{\ell} \times \mathcal{A}_{z}^{\kappa} .
$$

Due to the orthogonality of trigonometric functions, the above formula is none zero only when $n=\ell$ and $m=\kappa$ are satisfied. Therefore, the multiplex of the two coefficients, rather than the single, can be precisely calculated using (4). Furthermore, the relationship between $\varphi$ and $z$ in a helical periodic system is

$$
\begin{gathered}
\varphi=\varphi_{0}+\Delta \varphi, \\
\Delta \varphi=\Delta z \times \frac{2 \pi}{p} .
\end{gathered}
$$

Thus, the relationship between $\varphi$ and $z$ can be further presented as

$$
\varphi=\varphi_{0}+\left(z-z_{0}\right) \times \frac{2 \pi}{p},
$$

where $\varphi_{0}$ and $z_{0}$ are the initial coordinates. According to (8), there is a one-to-one match between $\varphi$ and $z$. Therefore, equation $\kappa=\ell$ must be true in (4). Equation (3) can therefore be 
(a)

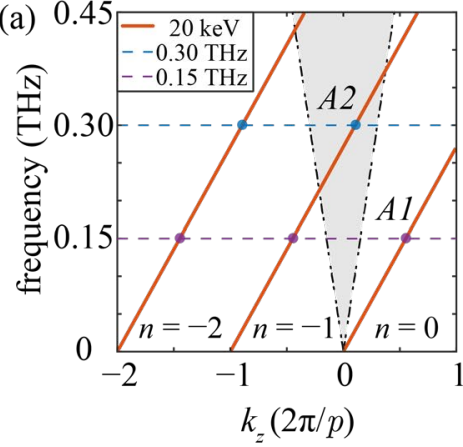

(d)

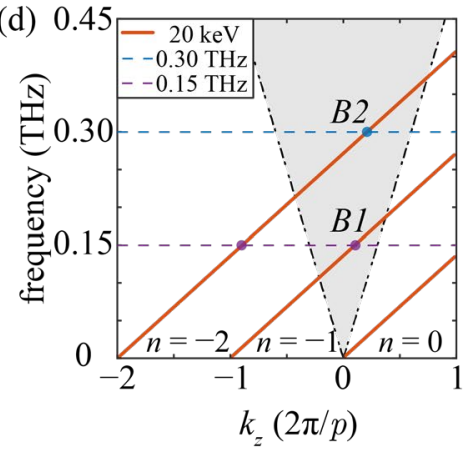

(b)

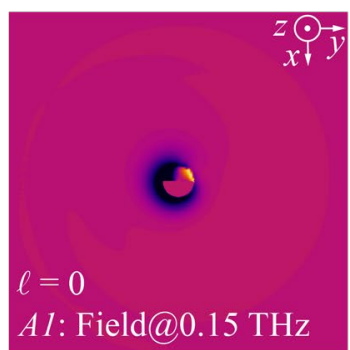

Min

(e)

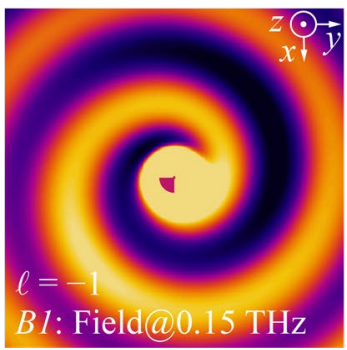

(c)

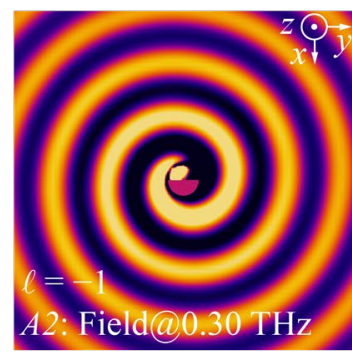

Max

(f)

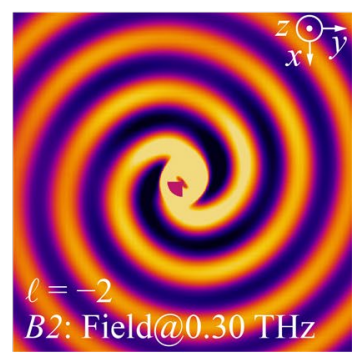

Fig. 2. (a), (b), and (c) belong to section-I of the helical grating. (d), (e), and (f) belong to section-II of the helical grating. (a) and (d) are Brillouin zone diagrams. (b) and (e) are profiles of $E_{z}$ at $0.15 \mathrm{THz}$. (c) and (f) are profiles of $E_{z}$ at $0.3 \mathrm{THz}$.

simplified by using $\kappa=\ell$ as

$$
u(\varphi, z)=\sum_{\ell=-\infty}^{\infty} \mathcal{A}_{\varphi}^{\ell} \mathcal{A}_{z}^{\ell} e^{j \ell \varphi} e^{j \ell \frac{2 \pi}{p} z} .
$$

Hence, the Fourier series of the $E_{z}(r, \varphi, z, t)$ can be obtained by substituting (9) into (1):

$$
E_{z}(r, \varphi, z, t)=R(r) \sum_{\ell=-\infty}^{\infty} \mathcal{A}_{\varphi}^{\ell} \mathcal{A}_{z}^{\ell} \times e^{j \ell \varphi} e^{-j\left(k_{z}-\ell \frac{2 \pi}{p}\right) z} e^{-j \omega t} .
$$

Equation (10) shows in the periodic system with helical modulation, the scattered field of the evanescent waves carried by the electron beam is superimposed by infinite plane waves with different amplitudes and wave vectors. Its $n$-th order spatial harmonic is:

$$
E_{z}^{n}(r, \varphi, z, t)=e^{j n \varphi}\left[R(r) \mathcal{A}_{\varphi}^{n} \mathcal{A}_{z}^{n} \times e^{-j\left(k_{z}-n \frac{2 \pi}{p}\right) z} e^{-j \omega t}\right] .
$$

According to (11), a phase factor $\exp (j n \varphi)$ is carried by the $n$-th spatial harmonic. Thus, the $n$-th order spatial harmonic carries OAM with $\mathrm{TC}=n$, which is induced by the interference of azimuthal and longitudinal Bloch waves in the helical periodic system. Similarly, in the left-hand helical periodic system, the $n$-th order spatial harmonic should carry OAM with $\mathrm{TC}=-n$. In the following, only the case of the right-hand helical periodic system is considered.

To verify the theoretical analysis, the double-section metallic helical grating is proposed and simulated by the particle-in-cell (PIC) solver in CST PARTICLE STUDIO [39], in which a 20-keV hollow electron beam is emitted from the left end of the structure [40]. To overcome the space-charge effect, a magnetic field of $0.5 \mathrm{~T}$ in the $z$-direction is also set. The dispersion characteristics of the electron beam in sections I and II are shown in Figs. 2(a) and 2(d) using the red line, and the gray region indicates the light cone. The electron beam first flies over section-I. As illustrated by the purple dashed line in Fig. 2(a), when the frequency is $0.15 \mathrm{THz}$, every spatial harmonic, including point $A 1$, locates in the slow-wave region. Accordingly, there should be no radiation waves at $0.15 \mathrm{THz}$, which is consistent with the profile of $E_{z}$ as shown in Fig. 2(b). However, when the frequency is $0.30 \mathrm{THz}$, according to the intersection point $A 2$, the $-1^{\text {st }}$ spatial harmonic locates in the light cone. Therefore, the $\mathrm{THz}$ vortex beam with $\mathrm{TC}=-1$ will radiate around the grating according to (11), and the profile shown in Fig. 2(c) proves the prediction. For section-II, the $-1^{\text {st }}$ and $-2^{\text {nd }}$ spatial harmonics are in the light cone at 0.15 and $0.3 \mathrm{THz}$, respectively, which are marked as $B 1$ and $B 2$ in Fig. 2(d). Thereby, the THz vortex beams with the TCs of -1 and -2 will be generated, which is also validated according to the electric field profiles shown in Figs. 2(e) and 2(f). And so forth, the $n$-th spatial harmonic can generate the vortex beams of $\mathrm{TC}=n$ in its corresponding radiation frequency region (the generation of higher-order TC is discussed in Section I of the Supplemental Material). As a result, using the helical periodic structure as shown in Fig. 1, a wideband $\mathrm{THz}$ vortex beam carrying arbitrary TCs can be generated by controlling the period of the pitch to construct the distribution of the spatial harmonic. However, this kind of SPR is incoherent, i.e., it will produce broad-spectrum radiation in all directions [41]. Based on SSPR, we will further study the $\mathrm{THz}$ vortex beams emitter with broad tuning bandwidth and tunable TCs. Moreover, it is worth noting that the generation of the vortex beams does not depend on the shape of the electron beam, a pencil beam can also produce this phenomenon. 
(a)
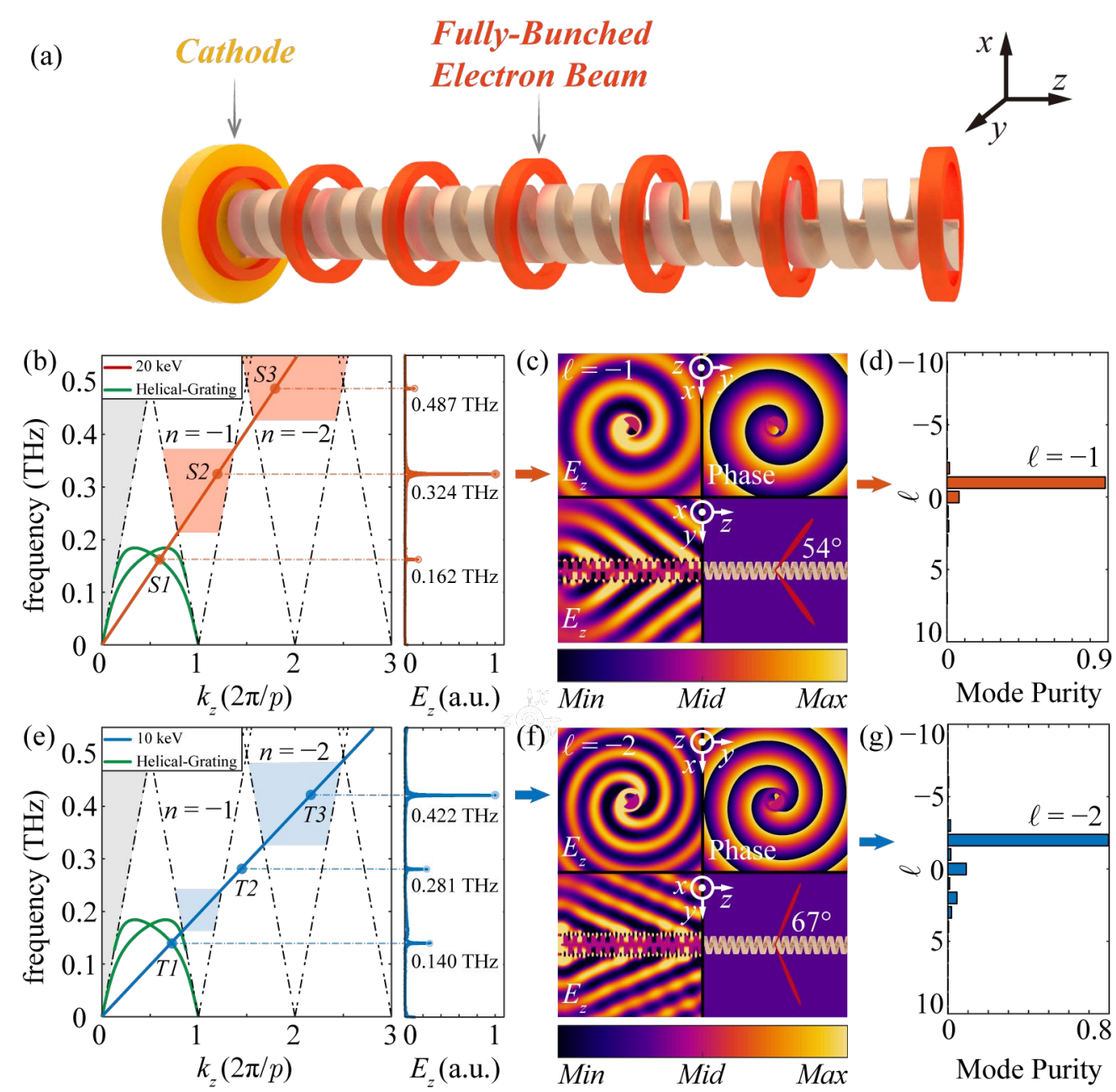

Fig. 3. (a) Schematic diagram of the vortex beam emitter. The structural parameters of the helical grating are the same as those of the section-I. When the energy of the electron beam is $20 \mathrm{keV}$ and $10 \mathrm{keV}$, the dispersion characteristics of helical grating and electron beam are shown in (b) and (e). The frequency spectrums of $E_{z}$ probed above the grating are shown in the right inset of (b) and (e). The profiles of $E_{z}$ are shown in (c) and (f), the results of mode purity for each vortex beam are illustrated by $(\mathrm{d})$ and $(\mathrm{g})$.

\section{VORTEX BEAM EMITTER BASED ON SSPR}

In this section, the helical grating integrated with the SSPR is utilized to emit the $\mathrm{THz}$ vortex beams with the $\mathrm{TC}$ of -1 or -2 . Firstly, the mechanism of harmonic-vorticity joint manipulation will be demonstrated. Spoof surface plasmon (SSP) are stimulated when the DC electron beam flies over the surface of the grating as shown in Fig. 3(a) [42]. If the beam-wave interaction between the electron beam and the SSP is sufficiently strong, the DC electron beam will be modulated and contains a certain percentage of AC components in the form of periodic bunches at the fixed frequency $f_{1}$, which is equal to that of the SSP, i.e., the frequency of intersection of the two dispersion lines as shown in Figs. 3(b) and 3(e). Therefore, the intensity of the scattered field of the evanescent waves carried by the electron beam will be greatly enhanced at the bunch frequency $f_{1}$ and $f_{m} . f_{m}$ is the harmonic frequency of the $f_{1}$ :

$$
f_{m}=m \times f_{1}, \quad m \in \mathbb{Z}^{+}, m \neq 1,
$$

where $m$ is an integer. If $f_{m}$ is in the radiation frequency region, the intensity-enhanced SSPR of $m$-th wave harmonic will be generated in a specific direction. The focus of the work presented in this paper is the range of the $n$-th spatial harmonic dispersion line that lies in the light cone. It is the point that the frequency of the $m$-th wave harmonic $\left(f_{m}\right)$ is confirmed by tuning that of the fundamental wave $\left(f_{1}\right)$. Once the $m$-th wave harmonic is in the range mentioned above, it can be determined that the $\mathrm{THz}$ vortex beam of $\mathrm{TC}=n$ is generated at the frequency of $f_{m}$. The analysis above corresponds to the proposed methodology of simultaneously manipulating the radiation frequency and the vorticity of the $\mathrm{THz}$ vortex.

To validate the concept, the configuration of the emitter with a fully bunched hollow electron beam is proposed and shown in Fig. 3(a). The helical grating structure is the same as the one shown in section-I, Fig. 1. Using numerical calculation methods (finite-differential time-domain) [39], the dispersion characteristics of the helical grating and electron beam are presented using green and red (blue) lines shown in Figs. 3(b) and 3(e) respectively. The intersection point of a $20 \mathrm{keV}$ electron beam dispersion line and the fundamental mode of the helical grating is marked as point $S 1$ in Fig. 3(b). The frequency of the electron bunch is $f_{S I}=0.162 \mathrm{THz}$, and the intensity of wave harmonics relating to $S 1$ will be greatly enhanced. Since 


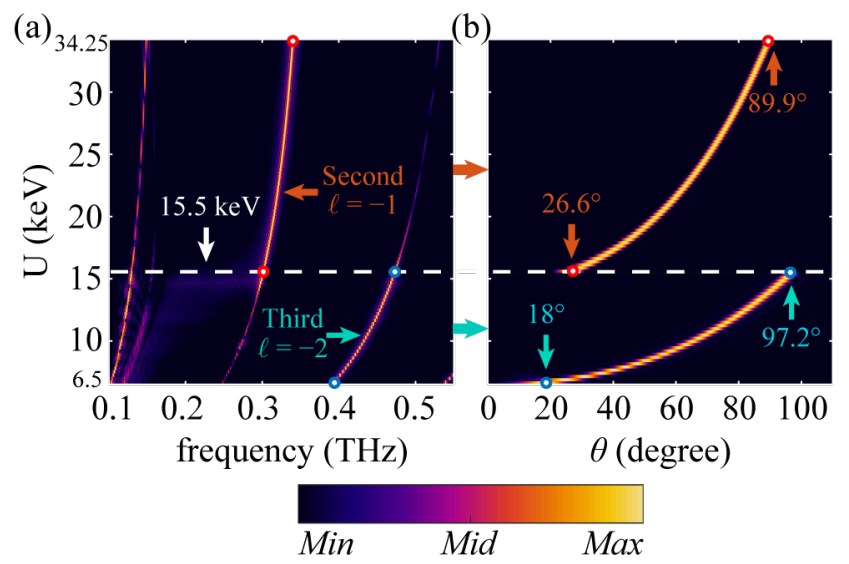

Fig. 4. As the energy of the electron is tuned, (a) shows the frequency spectra of $E_{z}$, and (b) shows the relations between the radiation intensity and the angle detected in the far-field.

the frequency of the second wave harmonic $f_{S 2}$ (point $S 2$ ) is located in the radiation region of $-1^{\text {st }}$ spatial harmonic (the pink region below), the SSPR vortex beam of $\mathrm{TC}=-1$ will be generated at $f_{S 2}=0.324 \mathrm{THz}$ according to (11). Similarly, the vortex beam of $\mathrm{TC}=-2$ will be generated at $f_{S 3}=0.487 \mathrm{THz}$. However, since the intensity of the second wave harmonic $S 2$ is much stronger than that of the third wave harmonic $S 3$ [43], the second harmonic vortex radiation at $f_{S 2}$ is dominant, which agrees well with the frequency spectrum of $E_{z}$ shown in the right inset of Fig. 3(b). This indicates the SSPR phenomenon. The profiles of $E_{z}$ at $f_{S 2}$ in this configuration are shown in Fig. 3(c). The phase and field distributions validate that the radiation wave is the vortex beam of $\mathrm{TC}=-1$. The radiation angle in the PIC simulation is $54^{\circ}$, which is consistent with the famous SPR relationship [34]:

$$
\lambda=\frac{L}{|n|}\left(\frac{1}{\beta}-\cos \theta\right)
$$

where $\lambda$ is the wavelength in free space, $\theta$ is radiation angle, $L$ is the period of the system, and $n$ is the harmonic order of SPR. Moreover, as shown in Fig. 3(d), the mode purity of the vortex beam at $f_{S 2}$ has also been calculated. The purity of the $\mathrm{TC}=-1$ component is as high as 0.88 , which proves its good performance (the detailed calculation method is discussed in Section II of the Supplemental Material). Such a pure OAM spectrum results from the radiation characteristics of SPR. Since the OAM is related to the order of the spatial harmonic, and the radiation interval between $-1^{\text {st }}$ and $-2^{\text {nd }}$ spatial harmonics is independent of each other, the TC of the generated vortex beam is tightly bonded to the radiation frequency region and will not overlap with other OAM modes. This demonstrates that the scheme operating at the second wave harmonic and $-1^{\text {st }}$ spatial harmonic can produce a pure vortex beam of $\mathrm{TC}=-1$. When the electron beam energy is $10 \mathrm{keV}$, the intersection point of its dispersion line and the lowest mode of the helical grating is $T 1$ shown in Fig. 3(e), and the bunch frequency is $f_{T I}=0.14 \mathrm{THz}$. Different from the previous configuration, the second wave harmonic $T 2$ carried by the $-1^{\text {st }}$ spatial harmonic is in the non-radiation region. Accordingly, the radiation at $f_{T 2}$ is suppressed. However, the third wave harmonic $T 3$ carried by
TABLE II

TUNABLE FEATURE

\begin{tabular}{lll}
\hline \hline $\mathrm{U}(\mathrm{keV})$ & {$[6.5,15.5]$} & {$[15.5,34.25]$} \\
Wave Harmonic & Third & Second \\
Spatial Harmonic & -2 & -1 \\
$\mathrm{TC}$ & -2 & -1 \\
Frequency $(\mathrm{THz})$ & {$[0.3723,0.4652]$} & {$[0.3101,0.3484]$} \\
$\theta$ (degree) & {$[18.0,97.2]$} & {$[26.6,89.9]$} \\
\hline \hline
\end{tabular}

the $-2^{\text {nd }}$ spatial harmonic is in the radiation region [the upper blue zone as shown in Fig. 3(e)]. Therefore, a vortex beam with $\mathrm{TC}=-2$ will be generated at $f_{T 3}=0.42 \mathrm{THz}$, which is consistent with the profile and distribution of $E_{z}$ at $T 3$ shown in Fig. 3(f). The radiation angle of $67^{\circ}$ also agrees well with (13). The mode purity of the vortex beam at $f_{T 3}$ is also calculated shown in Fig. $3(\mathrm{~g})$. The purity of the $\mathrm{TC}=-2$ component is as high as 0.80 . In brief, the scheme operating at the third wave harmonic and $-2^{\text {nd }}$ spatial harmonic can produce a pure vortex beam of $\mathrm{TC}=-2$.

Moreover, since the system operates on the wave harmonics of the SSPs of the helical grating, by altering the electron energy, the frequency of the wave harmonics will be tuned accordingly. To further study the tuneability, the frequency spectrums of $E_{z}$ and the far-field radiation characteristics are simulated when the electron energy is altered from 6.5 to $34.25 \mathrm{keV}$. The results are shown in Figs. 4(a) and 4(b). Similar to the analysis of $f_{S I}$ and $f_{T I}$, the prebunched frequency of the electron beam is equal to the intersection frequency in the simulation. When the electron energy is tuned between 6.5 and $15.5 \mathrm{keV}$, the vortex beam of $\mathrm{TC}=-2$ can be generated within $0.37-0.47 \mathrm{THz}$, which is induced from the radiation of the third wave harmonic carried by $-2^{\text {nd }}$ spatial harmonic. The radiation angle can be tuned from $18^{\circ}$ to $97.2^{\circ}$ accordingly. When the electron energy is tuned between 15.5 and $34.25 \mathrm{keV}$, the vortex beam of $\mathrm{TC}=-1$ can be generated between $0.31-0.35 \mathrm{THz}$, which is induced from the radiation of the second wave harmonic carried by $-1^{\text {st }}$ spatial harmonic. The radiation angle can be tuned from $26.6^{\circ}$ to $89.9^{\circ}$ accordingly. The above relationships are summarized in Table II. In summary, by tuning the electron energy, the frequency, radiation angle, and vorticity of the generated vortex beam can be tuned in a broad tuning bandwidth. Furthermore, due to the harmonic operation of this scheme, the communality of wavelength and the device size can be declined, which results in a decreased fabrication complexity.

\section{DISCUSSION}

The proposed vortex beam emitter is verified by using a fully bunched hollow-shape electron beam, which can generate the $\mathrm{THz}$ vortex beam with tunable $\mathrm{TC}$ in a broad tuning range. The hollow electron beam can be effectively achieved by an electron gun with a hollow emission surface and appropriate magnetic constraints (more description of how to realize such a hollowshape electron beam is shown in Section III of the Supplemental Material). However, the fully bunched electron beam is challenging to be obtained in the experiment. In practice, the techniques based on photocathodes can be used to form such a 


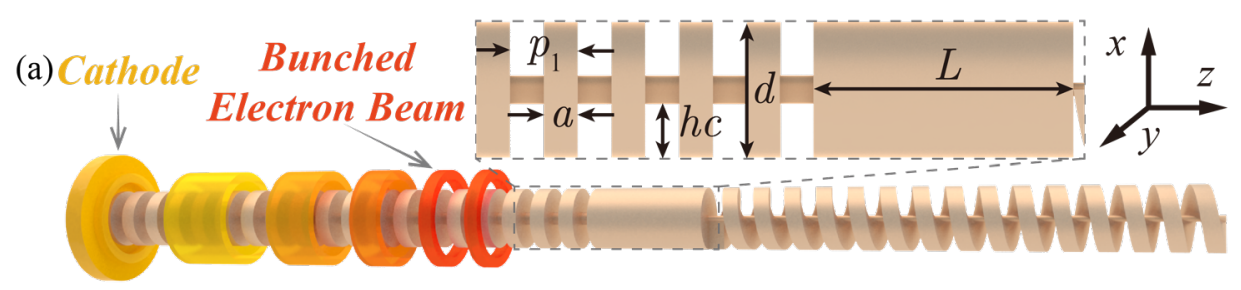

(b)
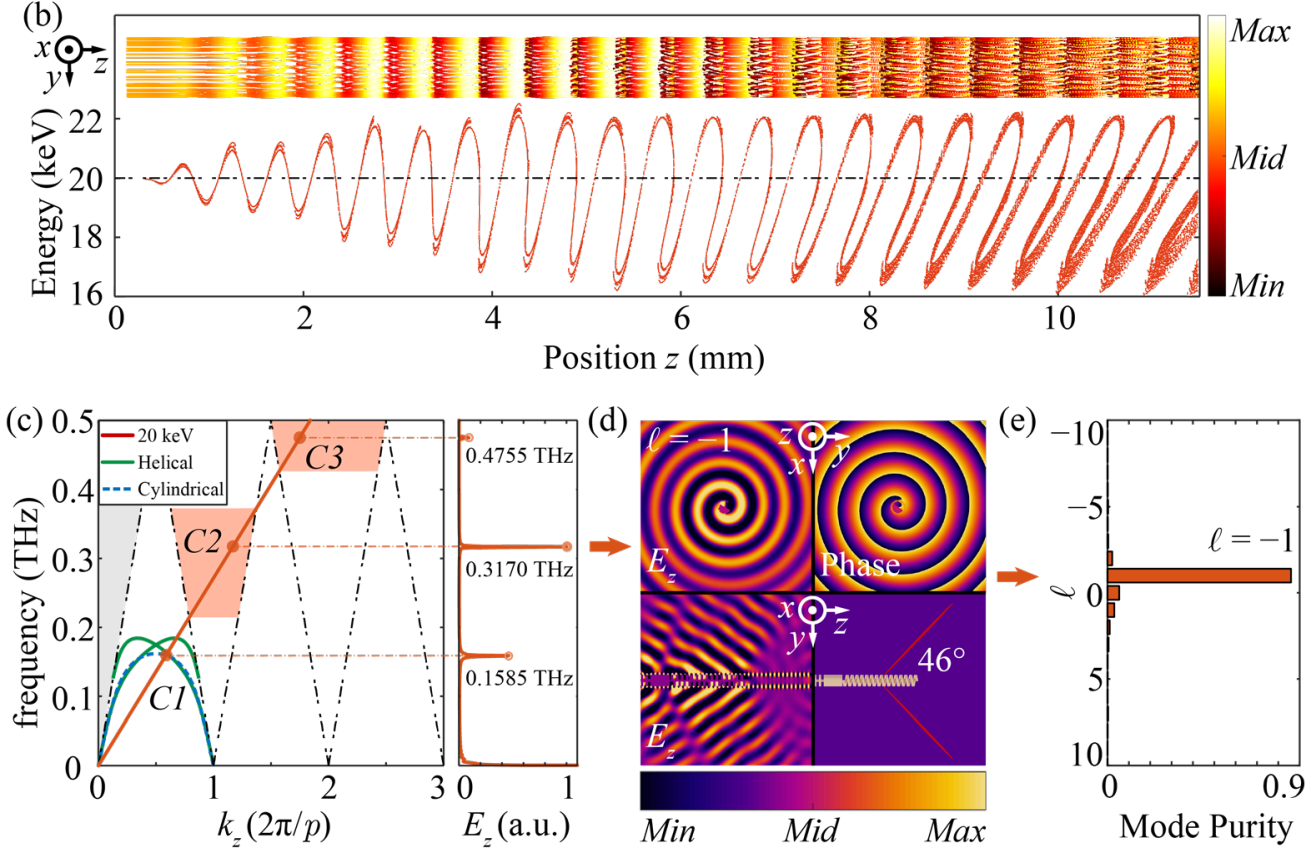

Fig. 5. (a) Schematic diagram of a prebunched electron beam flying over the surface of the metallic cylindrical grating and metallic helical grating. The parameters of the helical grating are the same as those of the section-I, and those of the cylindrical grating and the length of the drift region are shown in Table III. (b) The energy space distribution of the electron beam along the $z$-direction. When the energy of the electron beam is $20 \mathrm{keV}$ : (c) The dispersion relations of the electron beam, cylindrical grating, and helical grating. The right inset is the frequency spectrum of $E_{z}$. (d) Profiles of $E_{z}$ at $f_{C 2}=0.32 \mathrm{THz}$. (e) Mode purity of the vortex beam of $\mathrm{TC}=-1$.

beam [44]. In this paper, a section of the cylindrical grating is utilized to prebunch the DC electron beam to further facilitate the feasibility of the concept. The DC electron beam shown in Fig. 5(a) is firstly bunched around the cylindrical grating. Then, it interacts with SSP around the helical grating to generate the $\mathrm{THz}$ vortex beam. The dispersion relations of the $20-\mathrm{keV}$ electron beam, cylindrical grating [30], and helical grating are shown in Fig. 5 (c). The intersection point between the electron beam and the cylindrical grating is $\mathrm{Cl}$ with the frequency of $f_{C l}=0.16 \mathrm{THz}$. Therefore, when the DC electron beam first propagates over the surface of the cylindrical grating, the SSP of the grating will be excited at $f_{C l}$. The current density of the electron beam used here is sufficiently large $\left(150 \mathrm{~A} / \mathrm{cm}^{2}\right)$ to achieve a strong beam-wave interaction. Therefore, the DC electron beam can be effectively bunched at $f_{C l}$ as shown in Fig. 5(b) and would be further used to extract the $\mathrm{THz}$ vortex beam. When the well bunched hollow electron beam propagates over the helical grating, according to the analysis, the SSPR vortex beam of $\mathrm{TC}=-1$ and $\mathrm{TC}=-2$ will be generated at $f_{C 2}=0.32 \mathrm{THz}$ and $f_{C 3}=0.48 \mathrm{THz}$, respectively. Similarly, the radiation of the second wave harmonic is dominant corresponding to the frequency spectrum shown in the right inset of Fig. 5(c). In this case, the intensity of the fundamental wave is a bit stronger compared with that in the right inset of Fig. 3(b), which is due to the diffraction of the fundamental
TABLE III

PARAMETERS OF THE CYLINDRICAL GRATING AND DRIFT REGION

\begin{tabular}{ll}
\hline \hline Parameter & Size $(\mathrm{mm})$ \\
\hline$p_{1}$ & 0.30 \\
$a$ & 0.15 \\
$h c$ & 0.237 \\
$d$ & 0.60 \\
$L$ & 1.00 \\
\hline \hline
\end{tabular}

wave caused by the mismatch of the wave vector at the connection of the cylindrical grating and the helical one. The profile of $E_{z}$ at $f_{C 2}$ shown in Fig. 5(d) indicates that the radiation wave is the vortex beam of TC $=-1$, and the radiation angle $54^{\circ}$ agrees with (13). The mode purity of the vortex beam of $\mathrm{TC}=-1$ is 0.9 shown in Fig. 5(e), which reveals the good performance it maintains. By integrating the power flow density on a surface surrounding the helical grating, the average radiation power is $4 \mathrm{~W}$, and the efficiency of the system is about $0.25 \%$ (See Fig. S9(b) in Supplemental Material). To sum up, by using the cylindrical grating to efficaciously bunch the hollow electron beam, the proposed interaction circuit can effectively extract the SSPR vortex beam from the helical grating. Since dispersion characteristics of the cylindrical grating and helical grating are matched in a broad frequency 
range shown in Fig. 5(c) (blue and green lines), the tuning capability based on harmonic-vorticity joint manipulation in Section III could also be achieved by tuning the electron energy.

\section{CONCLUSIONS}

Efficient generation of the broadband $\mathrm{THz}$ vortex beam with tunable OAM is challenging but an appealing topic for many potential applications. In this paper, we demonstrate that the helical grating integrated with SSPR is capable of emitting THz vortex beams with controllable TCs. An explicit relation is established between the TC of the vortex beam and the index of spatial harmonics on the helical grating. Such a mechanism residing in the generation of OAM is induced by the azimuthal Bloch waves of the helical periodic system. By tuning the electron energy, the enhanced second or third wave harmonic of $-1^{\text {st }}$ or $-2^{\text {nd }}$ spatial harmonic can generate vortex beam of $\mathrm{TC}=-1$ or $\mathrm{TC}=-2$ and achieve a broad frequency tuning region in the $\mathrm{THz}$ band. Besides, a section of the cylindrical grating is utilized to effectively prebunch the electron beam toward successful harmonic excitation. The OAM purities of these vortex beams demonstrate their good performance which results from the SPR characteristic. Since the evanescent wave carried by the electron beam is broadband in nature, the proposed scheme can also be applied to microwave and mid-infrared bands.

\section{REFERENCES}

[1] L. Allen et al., "Orbital angular momentum of light and the transformation of Laguerre-Gaussian laser modes," Phys. Rev. A, vol. 45, no. 11, pp. 81858189, Jun. 1992.

[2] V. Y. Bazhenov et al., "Screw Dislocations in Light Wavefronts," J. Mod. Opt., vol. 39, no. 5, pp. 985-990, May 1992.

[3] G. Indebetouw, "Optical Vortices and Their Propagation," J. Mod. Opt., vol. 40, no. 1, pp. 73-87, Jan. 1993.

[4] S. Fürhapter et al., "Spiral phase contrast imaging in microscopy," Opt. Express, vol. 13, no. 3, pp. 689-694, Feb. 2005.

[5] K. Dholakia et al., "Shaping the future of manipulation," Nat. Photonics, vol. 5, no. 6, pp. 335-342, Jun. 2011.

[6] M. Padgett et al., "Tweezers with a twist," Nat. Photonics, vol. 5, no. 6, pp. 343-348, Jun. 2011.

[7] A. E. Willner et al., "Optical communications using orbital angular momentum beams," Adv. Opt. Photonics, vol. 7, no. 1, pp. 66-106, Mar. 2015.

[8] Y. Zhao et al., "Chirality detection of enantiomers using twisted optical metamaterials," Nat. Commun., vol. 8, no. 1, p. 14180, Jan. 2017.

[9] K. Humphreys et al., "Medical applications of terahertz imaging: a review of current technology and potential applications in biomedical engineering," in The 26th Annual International Conference of the IEEE Engineering in Medicine and Biology Society, Sept. 2004, vol. 1, pp. 1302 1305.

[10]E. A. Nanni et al., "Terahertz-driven linear electron acceleration," Nat. Commun., vol. 6, no. 1, p. 8486, Oct. 2015.

[11] T. L. Cocker et al., "Terahertz imaging with ultimate resolution," in 2016 Progress in Electromagnetic Research Symposium (PIERS), Aug. 2016, pp 3339-3339.

[12]A. A. Sirenko et al., "Terahertz Vortex Beam as a Spectroscopic Probe of Magnetic Excitations," Phys. Rev. Lett., vol. 122, no. 23, p. 237401, Jun. 2019.

[13]P. Yang et al., "6G Wireless Communications: Vision and Potential Techniques," IEEE Netw., vol. 33, no. 4, pp. 70-75, Jul. 2019.

[14]K. Miyamoto et al., "Direct observation of the topological charge of a terahertz vortex beam generated by a Tsurupica spiral phase plate," Appl. Phys. Lett., vol. 104, no. 26, p. 261104, Jul. 2014.

[15]H. Zhou et al., "Generation of Terahertz Vortices Using Metasurface With Circular Slits," IEEE Photonics J., vol. 6, no. 6, pp. 1-7, Dec. 2014.
[16]R. Imai et al., "Generation of broadband terahertz vortex beams," Opt. Lett., vol. 39, no. 13, pp. 3714-3717, Jul. 2014.

[17]N. Yu et al., "Light Propagation with Phase Discontinuities: Generalized Laws of Reflection and Refraction," Science, vol. 334, no. 6054, pp. 333337, Oct. 2011.

[18]B. A. Knyazev et al., "Generation of Terahertz Surface Plasmon Polaritons Using Nondiffractive Bessel Beams with Orbital Angular Momentum," Phys. Rev. Lett., vol. 115, no. 16, p. 163901, Oct. 2015.

[19]S.-J. Ge et al., "Generating, Separating and Polarizing Terahertz Vortex Beams via Liquid Crystals with Gradient-Rotation Directors," Crystals, vol. 7, no. 10, p. 314, Oct. 2017.

[20]A. A. Dhaybi et al., "Terahertz vortex beam generation by infrared vector beam rectification," J. Opt. Soc. Am. B, vol. 36, no. 1, pp. 12-18, Jan. 2019.

[21]Q. Lin et al., "Generation of terahertz vortex pulses without any need of manipulation in the terahertz region," Opt. Lett., vol. 44, no. 4, pp. 887890, Feb. 2019.

[22]D. Schurig et al., "Metamaterial Electromagnetic Cloak at Microwave Frequencies," Science, vol. 314, no. 5801, pp. 977-980, Nov. 2006.

[23] W. Liu et al., "Special Smith-Purcell radiation from an open resonator array," New. J. Phys., vol. 16, no. 7, p. 073006, Jul. 2014.

[24]Z. Wang et al., "Manipulating Smith-Purcell Emission with Babinet Metasurfaces," Phys. Rev. Lett., vol. 117, no. 15, p. 157401, Nov. 2016.

[25]Y. Yang et al., "Maximal spontaneous photon emission and energy loss from free electrons," Nat. Phys., vol. 14, no. 9, pp. 894-899, Sept. 2018.

[26]L. Jing et al., "Polarization Shaping of Free-Electron Radiation by Gradient Bianisotropic Metasurfaces," Laser Photonics Rev., vol. 15, no. 4, p. 2000426, Feb. 2021.

[27]L. Jing et al., "Spiral Field Generation in Smith-Purcell Radiation by Helical Metagratings," Research, vol. 2019, p. 3806132, Feb. 2019.

[28]M. Wang et al., "Vortex Smith-Purcell radiation generation with holographic grating," Photonics Res., vol. 8, no. 8, pp. 1309-1315, Aug. 2020.

[29]D. Li et al., "Growth rate and start current in Smith-Purcell free-electron lasers," Appl. Phys. Lett., vol. 100, no. 19, p. 191101, Apr. 2012.

[30]W. He et al., "Generation of broadband terahertz radiation using a backward wave oscillator and pseudospark-sourced electron beam," Appl. Phys. Lett., vol. 107, no. 13, p. 133501, Sept. 2015.

[31]J. Urata et al., "Superradiant Smith-Purcell Emission," Phys. Rev. Lett., vol. 80, no. 3, pp. 516-519, Jan. 1998.

[32]H. L. Andrews et al., "Superradiant emission of Smith-Purcell radiation," Phys. Rev. Spec. Top.-Accel. Beams, vol. 8, no. 11, p. 110702, Nov. 2005.

[33]S. E. Korbly et al., "Observation of Frequency-Locked Coherent Terahertz Smith-Purcell Radiation," Phys. Rev. Lett., vol. 94, no. 5, p. 054803, Feb. 2005.

[34]Y.-M. Shin et al., "Superradiant terahertz Smith-Purcell radiation from surface plasmon excited by counterstreaming electron beams," Appl. Phys. Lett., vol. 90, no. 3, p. 031502, Jan. 2007.

[35]Y. Yin et al., "Enhanced Superradiant Smith-Purcell Radiation in a ThreeMirror Quasi-Optical Cavity," IEEE Trans. Plasma Sci., vol. 37, no. 6, pp. 1057-1061, Apr. 2009.

[36]W. Liu et al., "Free electron terahertz wave radiation source with twosection periodical waveguide structures," J. Appl. Phys., vol. 111, no. 6, p. 063107, Mar. 2012.

[37]L. Liang et al., "High-harmonic terahertz Smith-Purcell free-electron-laser with two tandem cylindrical-gratings," Opt. Express, vol. 25, no. 3, pp. 2960-2968, Feb. 2017.

[38]P. Zhang et al., "Enhanced THz Smith-Purcell radiation based on the grating grooves with holes array," Opt. Express, vol. 25, no. 10, pp. 1090110910, May 2017.

[39]All numerical calculations were done with CST STUDIO SUITE.

[40]L. Liang et al., "High-harmonic terahertz Smith-Purcell free-electron-laser with two tandem cylindrical-gratings," Opt. Express, vol. 25, no. 3, pp. 2960-2968, Feb. 2017.

[41]S. J. Smith et al., "Visible Light from Localized Surface Charges Moving across a Grating," Phys. Rev., vol. 92, no. 4, pp. 1069-1069, Nov. 1953.

[42]J. Zhu et al., "Free-Electron-Driven Multi-Frequency Terahertz Radiation on a Super-Grating Structure," IEEE Access, vol. 7, pp. 181184-181190, Aug. 2019.

[43]W. Liu et al., "Multicolor Terahertz Frequency Mixer Using Multibunching of Free-Electron Beams," Phys. Rev. Appl., vol. 10, no. 3, p. 034031 , Sept. 2018.

[44] V. Joshi et al., "Theoretical and simulation study of 'Comb' electron beam and THz generation," Nucl. Instrum. Methods Phys. Res., Sect. A, vol. 913, pp. 28-39, Jan. 2019. 